\title{
A MONTE CARLO MODEL FOR PREDICTING THE EROSION RATE OF EB PVD
}

\section{TBCs}

\author{
R.G Wellman and J.R Nicholls \\ School of Industrial and Manufacturing Sciences \\ Cranfield University \\ Bedford, MK 43 OAL \\ $U K$
}

Since the introduction of electron beam (EB) physical vapour deposition (PVD) thermal barrier coatings (TBCs) and their application to moving components in the hot gas stream, erosion has become a prime concern. EB PVD TBCs, due to their unique columnar microstructure are far more strain tolerant than their plasma sprayed (PS) counter parts and can thus be used under more exacting operating conditions. It is under these operating conditions that erosion of the coated components is of primary importance.

The main aim of this project was the development of a computer model capable of predicting the erosion rate of EB PVD TBCs under various different conditions relevant to engine operation. In order to do this it was first necessary to determine the erosion mechanisms of EB PVD TBCs as well as their mechanical properties. Steady state erosion and single impact studies together with SEM were used to determine the erosion mechanisms. While nano indentation techniques were used to obtain the hardness and the Young's Modulus of the EB PVD TBC.

All these findings were then used in the development of a Monte-Carlo type computational erosion model capable of predicting the erosive wear rate of EB PVD TBCs under various conditions. The model which 
has been developed is capable of predicting the erosion rate of EB PVD TBC to within 30\%, so long as the erosion falls within the defined mechanism. This can easily be checked against an erosion map which has been developed. This paper discusses the program and reports some results from running the program under various conditions.

\section{INTRODUCTION}

Thermal barrier coatings act to protect the coated components from the hot gases in a gas turbine engine. Degradation of the coating through oxidation of the bond coat and subsequent spallation of the TBC or via the stepwise removal of material due to solid particle impact, either by ingested particles or particles generated by the engine itself, can result in localised hot spots and subsequent damage to, or reduced service life of, the component.

The primary aim of this project was to develop a computer model, based on Monte Carlo statistics that would predict the erosion rate of EB PVD TBCs in order to provide design engineers with a usable tool for predicting component life. But before a computer program could be written the erosion mechanism of the system to be modelled had to be analysed and fully understood. Thus, the first stage of the project concentrated on examining eroded specimens, analysing crack depths and distributions and gaining an insight into the erosion mechanism of EB PVD TBCs. These findings have been reported in a previous paper [1].

Nicholls and co-workers[2-5] have done significant work on modelling erosion, looking specifically at the erosion of oxide scales at various temperatures. Having completed a number of single impact and continuous impact studies, they concluded that there were three main operating regimes. These were 'substrate dominated' erosion, 'scale modified' erosion, and 'scale dominated' erosion, which were all functions of temperature, particle loading and impact dynamics. Since the TBCs are nominally 150-200 
$\mu \mathrm{m}$ thick the substrate properties are unlikely to have a major influence on the erosion mechanism, placing this program of work in the 'scale' dominated regime.

When eroding TBCs, the first consideration is the type of coating being eroded, since APS and EB-PVD coatings have different microstructures and hence erosion mechanisms. In all cases, for TBCs, material loss is via the initiation and propagation of cracks, and hence the erosion mechanism can be classified as brittle. However, under certain conditions, namely when the temperature is above the erodent softening temperature, EB-PVD coatings can exhibit a "ductile" type erosion response to impact angle, i.e. the maximum wear rate occurs at impact angles near $30^{\circ}[6]$. This is because when the erodent softens, as in the case of $\mathrm{SiO}_{2}$, at low impact angles it is able to pull out fractured sections of the coating that would otherwise not be removed. In other words, brittle fracture of the coating still occurs, but with enhanced loss of material at glancing impact angles. Other important process parameters, which will need to be defined / taken into account for any model, include particle flux, velocity, shape, density and mechanical properties.

EB PVD TBCs have a unique columnar microstructure which not only act to improve their strain tolerance but also act to improve their erosion resistance. Investigations into the erosion mechanisms have ascertained that the erosive wear of EB PVD TBCs proceeds via the accumulation of damage, in the form of near surface (lateral type) cracks in a number of adjacent columns, prior to the removal of the tops of the fractured columns [1]. It was also observed that cracks initiated in one column do not propagate into the neighbouring columns, the columnar boundaries, which give EB PVD TBCs their strain tolerance, also act to improve their erosion resistance. Although cracks initiated in one column do not propagate into neighbouring columns this does not mean that a single impacting particle can only initiate cracks in one column. If the impacting particle interacts with more than one column it is quite feasible that the impact could initiate near surface cracking in a cluster of adjacent columns. The unique columnar microstructure of EB PVD TBCs is illustrated in Figure 1, where examples of near surface cracking can also be seen. It 
has also been observed that cracking can also initiate from the edge of a column and propagate into the column. These types of cracks usually initiate from growth dendrites, as illustrated in Figure 2, which can act as circumferential notch defects.

\section{THE COMPUTER MODEL}

The computer model was developed using Delphi and is purely a computational tool with a number of input parameters, mainly material and system properties, and a single output, being the calculated dimensionless erosion rate. A number of assumptions have been made which are discussed in detail in the relevant sections. In order to model the erosion it was necessary be able to 'visualise' an area containing a set number of columns that could be impacted and since it was more likely than not that any impacting particle would interact with more than one column it was necessary to define first order and second order neighbours.

\subsection{Size of the Model}

In order to facilitate modelling the erosion of EB PVD TBCs it was decided to approximate the shape of the columns as hexagons. This means that each column has six identical nearest neighbours. The model comprises of 43 columns and 'wraps around' such that the columns on the left edge are the nearest neighbours of the right edge and the top to the bottom. This is sufficiently large to cover cases where an impacting particle strikes more than one column. The model comprises of a centre column with four concentric rings of columns around it. The first has 6 columns the second has 12 columns, the third has 18 columns, a fourth concentric ring of only 6 equally spaced columns was needed to ensure that all the columns have 6 nearest neighbours and 12 second order neighbours when curving the model around. 


\subsection{Flow of the Program}

In order to keep the run time of the programme to a minimum the model generates a look up table of values for a random distribution of impacting particles. The lookup table includes a number of key values for a random selection of 1000 particles. The calculations for the particles include contact area, size of the plastic zone, maximum impact force and the critical velocity for that particular particle size. The model then uses these values to calculate the erosion rate.

Once the lookup table has been completed the programme picks a random particle from the table then randomly picks a column to impact and a position on the column to impact. This allows the model to determine the percentage of the impact that will be absorbed by the impacted column and its first and second order neighbours. Then it determines whether the columns are already cracked. If not, it will calculate the depth of the possible lateral crack having checked that the impact force was sufficient to initiate cracking. This is done for the impacted column and all its first and second order neighbours which share a proportion of the impact.

After each impact the programme checks whether any column and at least four first order neighbours have been cracked. If they have the model, sums up the material above the crack, for the central column and each of the nearest neighbours that have been cracked, and adds it to the total of the mass of material removed. It then resets the columns as not cracked and picks another random particle from the lookup table.

Due to the fact that previous work on the erosion of EB PVD TBCs, conducted at Cranfield [1,6-9], would be used to verify the model, certain information was coded into the program. This included the particle size range of both the silica and the alumina erodents used in the erosion testing, as well as the pertinent mechanical properties. Thus the program can be tested against measured erosion rates for either alumina 
or silica. The program provides a third option for the choice of erodent and allows the operator to select a particle size characterised by a mean and a standard deviation. The flow diagram for the model is presented in Figure 2.

\subsubsection{Determining the Contact Position for the Centre of Impact}

For each impacting particle a random column from the 37 main columns is chosen where the centre of impact will occur. Within the column a number of positions have been defined where the centre of the impact can occur, which will determine how the impact/contact area interacts with the neighbouring columns. This is extremely important as only those columns that have been impacted can be cracked. For each of these impact positions and for a number of different contact-footprint sizes the percentage of the contact area falling on a column and its nearest neighbours has been determined manually. This was done by superimposing different circles on a layout of columns and then measuring the percentage of the area that fell on the different columns. The results were entered into the model as a lookup table, which allows the program to distribute the impacting force between the columns that 'share' the impact.

\subsubsection{Determining the Contact Area and the Size of the Plastic Zone}

When modelling the erosion of brittle materials the depth of the impact zone, produced by the particle, is very important. Evans et al. [10] postulated that lateral fracture initiates within a zone that extends to the elastic-plastic interface at full penetration. Hence, in modelling the erosion of brittle materials, if the depth of the plastic zone can be calculated the amount of material loss per impact can then be determined.

Slikkerveer [11] determined the maximum depth of penetration, for spherical particles, on impact to be: 


$$
Z_{\max }=\left(\frac{5 \pi \rho V^{2} k}{4}\right)^{2 / 5}\left(\frac{R^{3}}{r^{1 / 2}}\right)^{2 / 5}
$$

which, combined with Hill's [12] equation relating the depth of impact to the size of the plastic zone, enables one to determine the depth of cracking on impact, should cracking occur. It can be shown [13] that the indentation volume, the volume of the indenter that penetrates the substrate, determines the global stress field around the indenter. In other words, identical plastic zone boundaries develop for spherical and Vickers pyramidal indentations of equal volume. If one then approximates the indented volume to be hemispherical (with equal volume), it is the volume of the indent that is important not the shape, Hill's [12] inflated hole theory can be used to give a relationship between the indent size and the size of the plastic zone. This relationship can be approximated by the power law given in equation 2 . This approximation of the indented volume to a hemispherical shape could possibly result in a slight over estimation of the depth of the plastic zone.

$$
\frac{b}{a}=\mu\left(\frac{E}{H}\right)^{y}
$$

$\mu$ and $\mathrm{y}$ are fitted constants, $\mathrm{E}$ and $\mathrm{H}$ are the Young's modulus and Hardness of the substrate respectively, while 'b' and 'a' are respectively the radius of the plastic zone and radius of the indented volume.

A slightly different approach was proposed by Slikkerveer [11] for angular particles. He assumed a constant indentation hardness and then equated the kinetic energy of an impacting particle $\left(U_{k}\right)$ to the plastic work done and obtained the following equation: 


$$
U_{k}=\int_{0}^{\delta m} P(\delta) d \delta=H \int_{0}^{\delta m} A_{\text {ind }}(\delta) d \delta=H \delta_{v o l}
$$

Where $\mathrm{P}(\delta)$ is the indentation force at a depth of $\delta, \delta \mathrm{m}$ is the maximum indentation depth and $\delta_{\mathrm{vol}}$ is the volume of the indent.

$$
\begin{aligned}
& \Rightarrow \delta_{v o l}=\frac{U_{k}}{H} \\
& \delta_{v o l}=\frac{U_{k}}{H}=\frac{\frac{1}{2} m V^{2}}{H}=\frac{\frac{1}{2}\left(\frac{4}{3} \pi r^{3} \rho\right) V^{2}}{H}
\end{aligned}
$$

As discussed earlier, it is the volume of the indent that is important and this can be equated to a hemispherical indent of radius 'a' such that: $\delta_{v o l}=\frac{2}{3} \pi a^{3}$.

Thus the radius of the plastic zone, $b$, can be expressed as:

$$
\begin{aligned}
& b=\left(\frac{3 m V^{2}}{4 \pi H}\right)^{\frac{1}{3}}\left(\mu\left(\frac{E}{H}\right)^{y}\right) \\
& \Rightarrow b \propto m^{\frac{1}{3}} V^{\frac{2}{3}} H^{-\frac{1}{3}}
\end{aligned}
$$

Equation 17 is similar to the predictive model proposed by Evans and Wilshaw [14]. Thus it is possible to determine the depth at which lateral cracking would occur should the impacting particle supply sufficient 
energy to initiate lateral cracking. By using Equations 1 and 2 combined with Equation 6 the program is able to calculate the depth within a column at which cracking will occur for both angular and spherical impacting particles.

\subsubsection{Critical Velocity to Initiate Lateral Cracking}

In a brittle erosion model it is necessary at some stage to determine whether an impacting particle will initiate cracking in the target material. Thus some form of threshold value for the impact is needed. It was decided in this model to use a velocity threshold.

When the tensile stresses around a plastic zone, caused by indentation, exceeds the fracture limit cracks will initiate. Slikkerveer[11] proposed that this initiation could be expressed as a threshold value of the indentation volume $\delta_{\mathrm{vol}}$. This can be transformed to an equation for the threshold energy for lateral fracture which in turn yields an equation for a critical velocity needed to initiate lateral fracture, which is given by:

$$
V_{c r i t, l}=105 \frac{E^{3 / 4} K_{I C}^{3}}{H^{13 / 4} \rho^{1 / 2} R^{3 / 2}}
$$

Since the model used to derive this equation predicted no influence of particle shape the above equation can be used to predict lateral cracking for both spherical and angular impacting particles. The important factors in equation 8 are the density and the radius terms, which in fact equate to a mass term. Thus equation 8 can also be written as: 


$$
V_{c r i t, l}=215 \frac{E^{3 / 4} K_{I C}^{3}}{H^{13 / 4} m^{1 / 2}} \quad-9
$$

It must be noted that this is a quantitative estimate of the threshold and is based on the crack length being equal to the size of the plastic zone. The critical velocity is dependent on the particle size and decreases as the particle size and density increase. Thus, in the model, for each impacting particle it is necessary to calculate the critical velocity at which it will cause lateral cracking and then determine whether the impacting particle is above or below this critical velocity.

Equation 9 allows one to simply use the mass of the impacting particle to calculate the critical velocity; however, it is still necessary to determine the percentage of the mass, of the impacting particle, that any particular column sees. In a cluster of columns impacted by a particle each column experiences a different load, dependent on the percentage of the contact area that is in contact with the column in question. Each column in the cluster is dealt with separately as if it had been impacted by a single particle with a mass calculated as a certain percentage of the mass of the impacting particle. The percentage used is the same as the percentage of the contact area that is in contact with the column. This then enables the program to determine whether cracking occurs in different columns for a particular impact, when the contact footprint interacts with more than one column. It is also possible to use the percentage contact area and the percentage mass of the impacting particle interacting with a particular column to determine the radius of a particle with an equivalent mass thus enabling the use of equation 8. There is a certain degree of error inherent in both of these methods which increases as the contact area decreases. But since the small contact areas are unlikely to cause cracking this error is not a major issue.

There is expected to be a certain degree of error in the critical velocity. However, as an error in this calculation only becomes important in the region where the critical velocity approaches the velocity of the 
impacting particle, errors in the critical velocity are thus expected to have a minor influence on the predicted erosion rate of the target.

\subsubsection{Depth of Cracking in Nearest Neighbours}

For the column in which the impact centres the crack depth is taken as the radius of the plastic zone. However, in the neighbouring columns this is not the case as the shape of the plastic zone is assumed to be hemi-spherical. This means that the maximum depth of the plastic zone in the neighbouring columns will be less than the maximum calculated radius of the plastic zone for that particular impact. Thus in neighbouring columns the depth of the lateral cracking is assumed to occur at the maximum depth of the plastic zone in that column, which is at the column boundary shared with the column in which the impact is centred.

If the distance from the centre of the impact to the column boundary is known and the radius of the plastic zone is know then it is possible to calculate the depth of the plastic zone in the neighbouring columns. The plastic zone does not extend into neighbouring columns unless the impacting particle also impacts the neighbouring columns, in other words if no portion of the impacting footprint lands on a neighbouring column then it is totally unaffected by that particular impact. The program incorporates a look up table of the distance from the centre of an impact to the column boundary for each first and second order neighbour, as a function of column radius. Thus the cracks, in the columns adjacent to the column in which the impact centred, are most likely to initiate from the column boundary and propagate into the column, while the cracking in the column in which the impact centred is likely to occur within the bulk of the column.

\subsubsection{Calculating the Volume of Material Removed Per impact}


When calculating erosion rates one needs to determine volume of material lost per impact event. In the case of material loss via the formation of lateral cracks in a continuum the volume can be estimated as the volume of the spherical cap with the radius of the length of the lateral crack and the depth of the plastic zone.

However, in the case of EB PVD TBCs this method can not be used due to the columnar microstructure of the coating. As discussed earlier, cracks do not propagate across column boundaries. Thus material loss is limited by the size of the column. In the case of EB PVD TBC erosion the volume of material that is available for removal after impact for each individual column is given as:

$$
\text { vol }=3 *(\text { colradius })^{2} * \sin 60 * l
$$

Where column radius is the length of one side of the hexagon and 1 is the depth of the lateral crack, which is assumed to be the same as the depth of the plastic zone. The program uses Equation 10 to determine the volume loss of a column that has been cracked.

\subsubsection{Removal of Fractured Sections}

Two methods were used to determine the number of columns that need to be fractured before material is lost from the coating. The first was the analysis of the number of adjacent cracks in cross sectional views of an eroded sample and the other was the analysis of the top section of eroded samples before steady state erosion was reached [1]. On first analysis it appeared as though the central column and all its first order neighbours needed to be cracked in order for material to be removed from the surface of the coating. However, further analysis of the top section of a number of eroded samples revealed that only four or five nearest neighbours around the central column needed to be cracked for material to be lost from the 
columns. The model operates on the assumption that the central column and four nearest neighbours need to be cracked for material loss to occur.

\subsubsection{Input Parameters, Variables and Possible Limitations}

In order to make the computer program as versatile as possible, it has been designed such that all the input parameters that the program requires can be input by the operator. However, on compiling, the program does pre-select the parameters for silica as the erodent.

It must be noted that the model has no inherent limits set and thus will calculate values according to the input parameters. Thus it is up to the operator to ensure that the input values are reasonable and that the regime under investigation is compatible with the model upon which the program is based, i.e. near surface cracking and no gross deformation of the coating.

Erosion maps are an excellent way to determine the erosion regime in which the system is operating. To this end an erosion map was constructed for the erosion of EB PVD TBCs with silica [9], the map has subsequently been modified to include an intermediate damage mechanism, termed compaction damage, which occurs between erosion and foreign object damage, as illustrated in Figure 4. Such an erosion map allows one to determine, from the particle size and the impact velocity, whether or not the erosion mechanism will be in the correct regime for the model to generate meaningful results.

\section{RESULTS FROM THE MODEL AND DISCUSSION}

The model was run under various conditions the results of which are represented in a number of graphs and tables in the following sections. Each point on a graph is an average of 5 repeat runs. This was done because of the natural scatter in results generated by the statistical methods used in the program. These 
results, except where otherwise stated, were all generated using the particle size distributions that were coded into the program and were based on the particle size distribution of the erodents that were used by Jaslier[7]. This was done so that the model could be validated from the experimental results that Jaslier obtained from erosion testing[6-8].

For reasons of speed only $0.01 \mathrm{~g}$ of erodent was used to determine the erosion rate for each test. If more erodent was used the run time of the program became excessive. However, some tests were run with more erodent and there was essentially no difference in the dimensionless erosion rates obtained.

Unless otherwise stated the graphs were all generated using the input parameters outlined in Table 1 and assuming that the central column and 4 nearest neighbours need to have fractured for material loss to occur. A shape factor of unity was used for silica as erosion data to which the program results have been compared was generated using spherical silica as the erodent.

\subsection{Repeatability of the Model}

As can be seen from the low standard deviations in Table 2 the repeatability of the model is very good and is in the order of a few percent. However, there appears to be an increase in the standard deviation with an increase in erosion rate.

\subsection{Validation of the Model}

In order to validate the model, the program was run under the same conditions as previous experimental tests conducted at Cranfield University[6-8], which used silica with an average radius of $30 \mu \mathrm{m}$ as the erodent at a velocity of $170 \mathrm{~m} / \mathrm{s}$ at room temperature. As can be seen from Table 3, under these conditions, there is a good correlation between the predicted erosion rates and the measured erosion rates. 
Further the literature[6] reports a linear increase in erosion rate with velocity in the zero to $300 \mathrm{~m} / \mathrm{s}(0-230$ for alumina) velocity range with $\mathrm{E}_{\text {rate }}=\mathrm{cV}$, where $\mathrm{c}$ is 0.102 for silica at room temperature. The predicted erosion rates are near linear in this region with a value for $\mathrm{c}$ of 0.122 . With alumina as the erodent $\mathrm{c}$ is 0.131 experimentally, while the predicted rates give $\mathrm{c}$ as 0.200 , which is significantly higher than that reported in the literature, or 0.148 if calculated from $0-230 \mathrm{~m} / \mathrm{s}$. The latter value agrees much better with the experimental data, which was determined for velocities up to $230 \mathrm{~m} / \mathrm{s}$ for alumina as the erodent.

It is obvious from the graph of velocity vs erosion rate (Figure 5) for both silica and alumina as the erodent, that there is not a true linear relationship between erosion rate and velocity. However, it was decided to assume a linear rate over the range $0-300 \mathrm{~m} / \mathrm{s}$ in order to calculate the values of ' $\mathrm{c}$ ' for the purpose of comparing the results to those reported in the literature. It is understandable that Yaslier et al[ 7,8$]$ assumed a linear relationship between erosion and velocity since the range of velocities over which they were able to test was limited, and over the range that they tested the coatings it appeared as though there was a linear relationship with velocity. However, the model was able to 'test' the coatings at higher velocities than could be obtained in the erosion rig, and the results show that erosion is not linear but in fact follows an exponential type relationship.

Erosion rate is often reported in the literature as:

$$
\mathrm{E}_{\text {rate }}=\mathrm{kV}^{\mathrm{n}}
$$

Where $\mathrm{k}$ is a constant, $\mathrm{V}$ is the velocity of impact and $\mathrm{n}$ has a value typically between 2 and 4 . For the erosion of an EB PVD TBC, the exponent is greater than one but less than 2 as indicated in Table 4. 
It is entirely possible that at lower impact angles inaccuracies will creep into the results due to the fact that it is probable that some of the horizontal component of the impacting velocity will be utilised in facilitating the removal of fractured sections of the coating and hence increase the erosion rate. Thus the model would predict a lower erosion rate than actually occurs in practice. It is also probable that angular particles would accentuate this phenomenon.

The graph in Figure 6 is a comparison between the predicted and the measured [6] erosion rates for an EB PVD TBC eroded with silica at $170 \mathrm{~m} / \mathrm{s}$ at various impact angles. As can be seen there is a good correlation between the two sets of results with the predicted results being within $20 \%$ of the measured results. If the model was $100 \%$ accurate all the points would fall on the solid line.

Davis et al[15] also reported results for EB PVD TBC coatings eroded with 63-130 $\mu \mathrm{m}$ silica at $30 \mathrm{~m} / \mathrm{s}$, which could be used to test the model. As can be seen from Table 5, there is a significant difference between the reported erosion rates of Davis et al [15] and those predicted by the model. There are a number of factors, which account for this major discrepancy in the predicted results. The most important of these being the size of the impacting particles, which was reported as being between $63-100 \mu \mathrm{m}$, but no size distribution was reported. Further, if one looks at the erosion map, due to the low velocity of $30 \mathrm{~m} / \mathrm{s}$ it appears as though the system is operating over the transition point of the lateral crack erosion mechanism. Thus the particle size distribution is extremely important as only the larger particles will cause damage. Other factors, which will affect the predicted erosion rates, are the hardness and the fracture toughness of the columns, but although the hardness of the coating is reported, there was no information on the properties of the individual columns. And finally the coating was a $20 \mathrm{wt} \% \mathrm{Y}_{2} \mathrm{O}_{3}$ and not an $8 \mathrm{wt} \% \mathrm{Y}_{2} \mathrm{O}_{3}-\mathrm{ZrO}_{2} \mathrm{~EB}$ PVD TBC.

The first set of predicted results in Table 5 was calculated using the input parameters coded into the model but with a mean particle size of $50 \mu \mathrm{m}$ (radius) STD $20 \mu \mathrm{m}$. The second set of results was calculated using 
the same particle size range but with a column diameter of $10 \mu \mathrm{m}$, the size of the columns was estimated from the micrograph in the reference [15]. The difference in the two sets of predicted erosion rates illustrates how important the input parameters are in obtaining accurate results from the model.

\subsection{Effect of Column diameter on the Erosion Rate}

The erosion of EB PVD TBCs does not follow a classic Hall Petch type relationship for column diameter vs erosion rate. The increase in erosion rate with the column size, shown in Figure 7, was expected as the program assumes that if a column is fractured the crack will run across the whole column. Thus an increase in the column size will result in an increase in the erosion rate of the material. Although in most cases this is true, as the column size increases a stage will be reached where the cracks will not always run across the whole column. At this point the program will start to over estimate the erosion rate.

\subsection{Effect of Particle Size on Erosion Rate}

In order to determine the effect of particle size on the erosion rate, the model was run under standard conditions for silica as the erodent except that the particle size was varied and the standard deviation for the particle size was taken as $1 \mu \mathrm{m}$. From Figure 8 it appears as though erosion rate initially increases with an increase in particle size to about $15 \mu \mathrm{m}$ and then decreases as the particle size increases. This result is partly due to the fact that the erosion rate is reported as mass loss per mass erodent, and is related to both the number of impacting particles and a threshold particle size.

The initial increase in the erosion rate with particle size is due to the fact that very small particles, particles less than $10 \mu \mathrm{m}$, do not cause cracking in the coating and hence the model predicts an erosion rate of zero. After this threshold is passed the dimensionless erosion rate increases with an increase in particle size. As the particles increase further in size the number of particles per unit mass reduces faster than the 
size of the damage zone increases due to the increase in particle size, thus the overall result is a decrease in the dimensionless erosion rate as the particle size increases. Under normal erosion conditions, i.e. when eroding a continuum, the larger particles will cause considerably more damage than the smaller ones and hence the dimensionless erosion rate will increase with particle size, to about $100 \mu \mathrm{m}$, after which a further increase in particle size does not result in an increase in the dimensionless erosion rate. This increase in erosion with particles size is attributed to the larger crack lengths generated by the larger particles, and hence a larger amount of material is available for material removal. However, due to the columnar microstructure of the EB PVD TBC, the length of the crack generated by an impacting particle is limited by the size of the column, since the cracks do not propagate across the column boundaries. Thus in the erosion of EB PVD TBCs it is the number of impacts which cause cracking that is the rate-limiting factor and not the length of the crack generated, as is the case for a continuum. It must be noted that an increase in the particle size results in an increase in the contact area which can result in an increase in the number of columns damaged per impact, depending on the particle size, the column diameter and the impact velocity. But this of cause is offset by the reduction in the number of impacting particles.

Another way of showing the effect of particle size on the erosion rate is to plot erosion rate per impact against the particle size of the impacting erodent. This is illustrated in Figure 9 and as can be seen the erosion rate increases with an increase in the particle size. A trend line has been added to the graph and forced through the intercept. The plotted data diverges from the trend line at the lower particle sizes due to the threshold effects.

\subsection{Effect of EB PVD TBC Hardness on the Erosion Rate}

At first glance the effect of the target hardness, Figure 10, seems to be anomalous. However, it must be noted that the hardness of the target is used in two separate sections of the program with counteracting results. In the first of these the target hardness is used in the calculation of the depth of the plastic zone. 
In this case as the hardness increases the depth of penetration decreases so the erosion rate decreases. However, the hardness of the target is also used in determining the critical velocity for crack initiation. In this case as the hardness increases the critical velocity to initiate cracking decreases so the erosion rate increases. This is the dominant relationship until the hardness increases to the stage where most of the impacting particles have sufficient velocity to initiate cracking. After this stage is reached the reduction in the size of the impact event due to an increase in the hardness becomes the dominant relationship between target hardness and erosion rate and hence the erosion rate decreases as the target hardness increases.

However, if it were possible to decrease the hardness of the EB PVD TBC to below 9 GPa it is entirely possible that a different erosion mechanism, namely one entailing damage accumulation, would be operating, which would require modification of the current model.

\subsection{Temperature}

The affect of temperature on the erosion of TBCs and other materials is highly complicated and system dependent. As such the program is not designed to operate at elevated temperatures. However, in certain cases as with TBCs eroded by alumina, where the erosion mechanism is not affected by the increase in the temperature, the model can still be used to predict the erosion rate. This is assuming that the operator knows how the material properties of the erodent and the target change with temperature and is able to enter the correct values into the program.

\section{CONCLUSIONS}

1. A model has been developed that permits the prediction of the erosion rate of EB PVD TBCs under a wide range of erosion conditions. 
2. The program has been shown to be accurate to within $\pm 30 \%$, in some cases $\pm 10 \%$ for this wide range of operating conditions.

3. The erosion rate of EB PVD TBCs is not linear as previously reported, but follows a velocity power law $\mathrm{E}_{\text {rate }}=\mathrm{kV}^{\mathrm{n}}$, where $\mathrm{n}=1.29$ for silica and 1.51 for alumina.

4. Reducing column diameter significantly reduces the erosion rate.

5. The erosion rate of EB PVD TBCs could be reduced by reducing the size of the dendrites formed during the deposition process, as these behave like a circumferential notch, lowering the stress needed to cause fracture.

6. The model shows a better correlation with the results from literature using silica as the erodent than using alumina as the erodent.

7. The model is capable of predicting how erosion rate will change with changes in certain material properties. 


\section{REFERENCE LIST}

1. R.G Wellman and J.R Nicholls, Wear, 242, (2000) 89.

2. D.J Stephenson and J.R Nicholls, Corrosion Science, 35, (1993) 1015.

3. D.J Stephenson and J.R Nicholls, Wear, 186-187, (1995) 284.

4. J.R Nicholls and D.J Stephenson, Wear, 186-187, (1995) 64.

5. P Hancock, J.R Nicholls and D.J Stephenson, Surface and Coatings Technology, 32, (1987) 285.

6. J.R Nicholls, Y Jaslier and D.S Rickerby, 4th International Colloquia on Corrosion and Protection of Materials at High Temperatures, Les Embies France, 1996.

7. Y Jaslier, PhD Thesis, Cranfield University (1995).

8. J.R Nicholls, Y Jaslier and D.S Rickerby, Materials at High Temperature, 15, (1998) 15.

9. R.G Wellman and J.R Nicholls, Euromat 2001, Rimini Italy, 2001.

10. A.G Evans, M.E Gulden and M Rosenblatt, Proceedings of the Royal Society (1978) 343.

11. P.J Slikkerveer, Mechanical Etching of Glass by Powder Blasting, Eindhoven, 1999.

12. R Hill, Plasticity, Oxford University Press, Oxford, 1950.

13. S.S Chiang, D.B Marshall and A.G Evans, Journal of Applied Physics, 53, (1982) 298.

14. A.G Evans and T.R Wilshaw, Acta Metallurgica, 24, (1976) 939.

15. A.G Davis, D.H Boone and A.V Levy, Wear, 110, (1986) 101. 
NOMENCLATURE

\begin{tabular}{|l|l|}
\hline$A$ & Radius of indented volume \\
\hline$A_{\text {ind }}$ & Area of indent \\
\hline$b$ & Radius of plastic zone \\
\hline$E$ & Young's modulus \\
\hline$E_{\text {rate }}$ & Erosion rate \\
\hline$H$ & Hardness \\
\hline$k$ & Constant \\
\hline$K_{I C}$ & Fracture toughness of the column \\
\hline 1 & Depth of lateral cracking \\
\hline$m$ & Mass of impacting particle \\
\hline$n$ & Rate exponential \\
\hline$P$ & Indentation force \\
\hline$R$ & Particle radius \\
\hline$r$ & Radius of contact point \\
\hline$U_{k}$ & Kinetic energy of erodent particle \\
\hline$V$ & Particle Velocity \\
\hline$Y$ & Constant \\
\hline$Z$ & Depth of penetration \\
\hline$\delta_{\text {vol }}$ & Volume of indent \\
\hline
\end{tabular}


Table 1: Table of input parameters used in running the program.

Table 2: Table of erosion rate and standard deviation for a number of conditions.

Table 3: Table of results of Model compared to results from the literature for erosion with silica at $170 \mathrm{~m} / \mathrm{s}$ and various impact angles. *Acknowledged by the Authors as an unusually high result.

Table 4: Values of ' $k$ ' and ' $n$ ' for silica and alumina as the erodents.

Table 5: Comparison of predicted erosion rates vs. results in the literature.

Figure 1: Micrograph of the cross section of an eroded EB PVD TBC showing the columnar microstructure and near surface cracking.

Figure 2: SEM micrograph showing a crack which has initiated from a growth dendrite and propagated into the column [1].

Figure 3: Flow diagram for the EB PVD TBC erosion model. Where ' $\mathrm{H}_{\mathrm{r}}$ ' is the contact radius, ' $\mathrm{F}_{\max }$ ' is the maximum impacting force of the particle, ' $\mathrm{F}_{\text {crit }}$ is the critical force necessary to initiate cracking in the column and ' $\mathrm{NN}$ ' is the nearest neighbour.

Figure 4: Erosion map for EB PVD TBC's eroded with silica.

Figure 5: Effect of impact velocity on the erosion rate of EB PVD TBCs.

Figure 6: Graph showing the comparison between predicted and measured [6] erosion rates (the superimposed line is drawn for a direct comparison the dashed lines represent one standard deviation).

Figure 7: The effect of column diameter on the erosion rate of EB PVD TBCs eroded with silica.

Figure 8: Effect of particle size on the erosion rate of EB PVD TBCs eroded with silica.

Figure 9: Erosion rate as a function of particle size.

Figure 10: Effect of coating hardness on the erosion rate. 


\begin{tabular}{|l|l|l|}
\hline Parameter & $\mathbf{S i O}_{2}$ & $\mathbf{A l}_{2} \mathbf{O}_{3}$ \\
\hline Poisson's Ratio of Target & 0.22 & 0.22 \\
\hline Young's Modulus of Target & $200 \mathrm{GPa}$ & $200 \mathrm{GPa}$ \\
\hline Particle Density & $2600 \mathrm{~kg} / \mathrm{m}^{3}$ & $3900 \mathrm{~kg} / \mathrm{m}^{3}$ \\
\hline Poisson's Ratio of Particle & 0.22 & 0.22 \\
\hline Young's Modulus of Particle & $94 \mathrm{GPa}$ & $390 \mathrm{GPa}$ \\
\hline Mass of Erodent & $0.01 \mathrm{~g}$ & 0.01 \\
\hline Fracture Toughness of the Target & $2.3 \mathrm{MNm}$ & $2.3 / 2$ \\
\hline Hardness of the Target & $14 \mathrm{GPa}$ & $14 \mathrm{GPa}$ \\
\hline Mean Particle size of the Erodent & $30 \mu \mathrm{m}$ & $50 \mu \mathrm{m}$ \\
\hline Erodent Shape particle if & round & sharp \\
\hline $\begin{array}{l}\text { Shape Factor of the } \\
\text { Rounded }\end{array}$ & 1 & - \\
\hline
\end{tabular}

Table 1: Table of input parameters used in running the program. 


\begin{tabular}{|l|ll|l|l|l|l|}
\hline Erodent & & & $100 \mathrm{~m} / \mathrm{s}$ & $200 \mathrm{~m} / \mathrm{s}$ & $300 \mathrm{~m} / \mathrm{s}$ & $400 \mathrm{~m} / \mathrm{s}$ \\
\hline \multirow{3}{*}{ silica } & Erosion rate & $(\mathrm{g} / \mathrm{kg})$ & 9.63 & 20.85 & 37.05 & 57.77 \\
\cline { 2 - 7 } & STD & $(\mathrm{g} / \mathrm{kg})$ & 0.14 & 0.43 & 0.43 & 1.65 \\
\hline \multirow{2}{*}{ Alumina } & Erosion rate $(\mathrm{g} / \mathrm{kg})$ & 10.02 & 30.90 & 59.83 & 82.30 \\
\cline { 2 - 7 } & STD & $(\mathrm{g} / \mathrm{kg})$ & 0.19 & 0.52 & 0.42 & 1.05 \\
\hline
\end{tabular}

Table 2: Table of erosion rates and standard deviations for a number of conditions calculated by the model. 


\begin{tabular}{|l|l|l|l|l|}
\hline $\begin{array}{l}\text { Impact Angle } \\
\left({ }^{\circ}\right.\end{array}$ & $\begin{array}{l}\text { Erosion Rate }(\mathrm{g} / \mathrm{kg}) \\
\text { From Model }\end{array}$ & STD & $\begin{array}{l}\text { Erosion Rate }(\mathrm{g} / \mathrm{kg}) \\
\text { From Literature [6] }\end{array}$ & $\%$ Difference \\
\hline 90 & 16.52 & 0.33 & 17.5 & 5.9 \\
\hline 75 & 16.14 & 0.31 & 19.5 & 20.8 \\
\hline 60 & 14.15 & 0.13 & 13.8 & 2.5 \\
\hline 45 & 11.22 & 0.20 & $31.2^{*}$ & - \\
\hline 30 & 8.27 & 0.04 & 10 & 20.9 \\
\hline
\end{tabular}

Table 3: Table of results of Model compared to results from the literature for erosion with silica at $170 \mathrm{~m} / \mathrm{s}$ and various impact angles. *Acknowledged by the Authors as an unusually high result. 


\begin{tabular}{|l|l|l|}
\hline & Silica & Alumina \\
\hline $\mathrm{k}$ & $2.51 \mathrm{E}-2$ & $9.91 \mathrm{E}-3$ \\
\hline $\mathrm{n}$ & 1.29 & 1.51 \\
\hline
\end{tabular}

Table 4: Values of ' $k$ ' and ' $n$ ' for silica and alumina as the erodents. 


\begin{tabular}{|l|l|l|}
\hline & $30^{\circ}$ & $90^{\circ}$ \\
\hline Literature $(\mathrm{g} / \mathrm{kg})$ & 0.12 & 0.16 \\
\hline Model Prediction A (g/kg) & 0.22 & 0.84 \\
\hline Model Prediction B (g/kg) & 0.094 & 0.37 \\
\hline
\end{tabular}

Table 5: Comparison of predicted erosion rates vs. results in the literature from Davis et al [15]. 


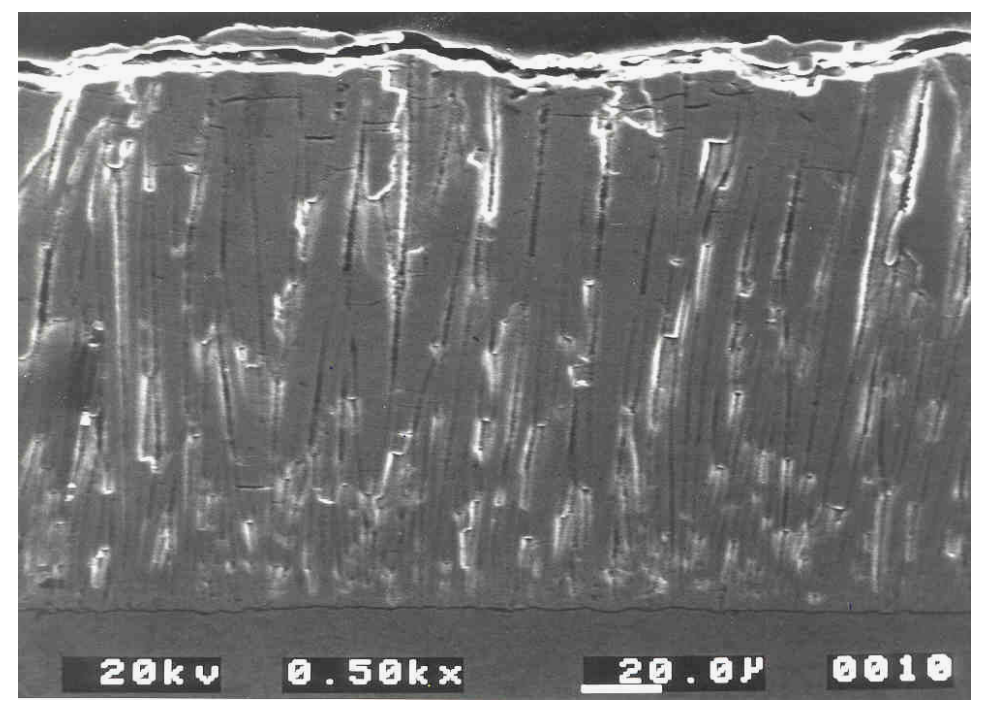

Figure 1: Micrograph of the cross section of an eroded EB PVD TBC showing the columnar microstructure and near surface cracking. 


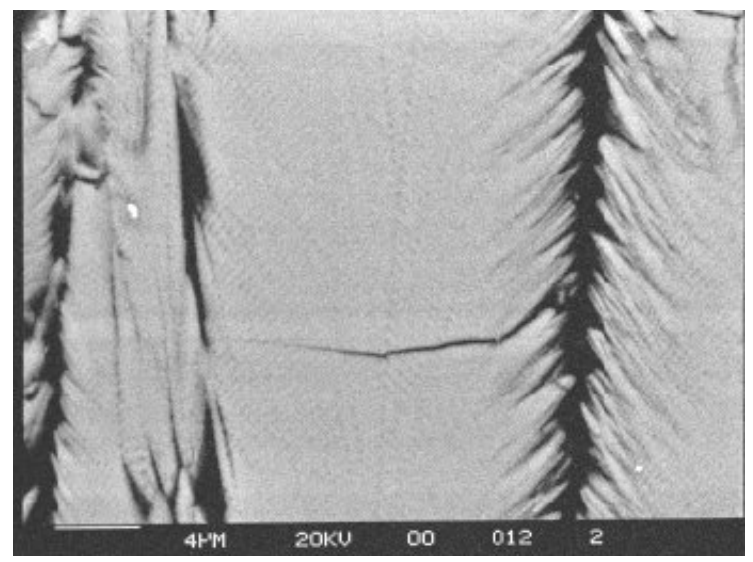

Figure 2: SEM micrograph showing a crack which has initiated from a growth dendrite and propagated into the column [1]. 


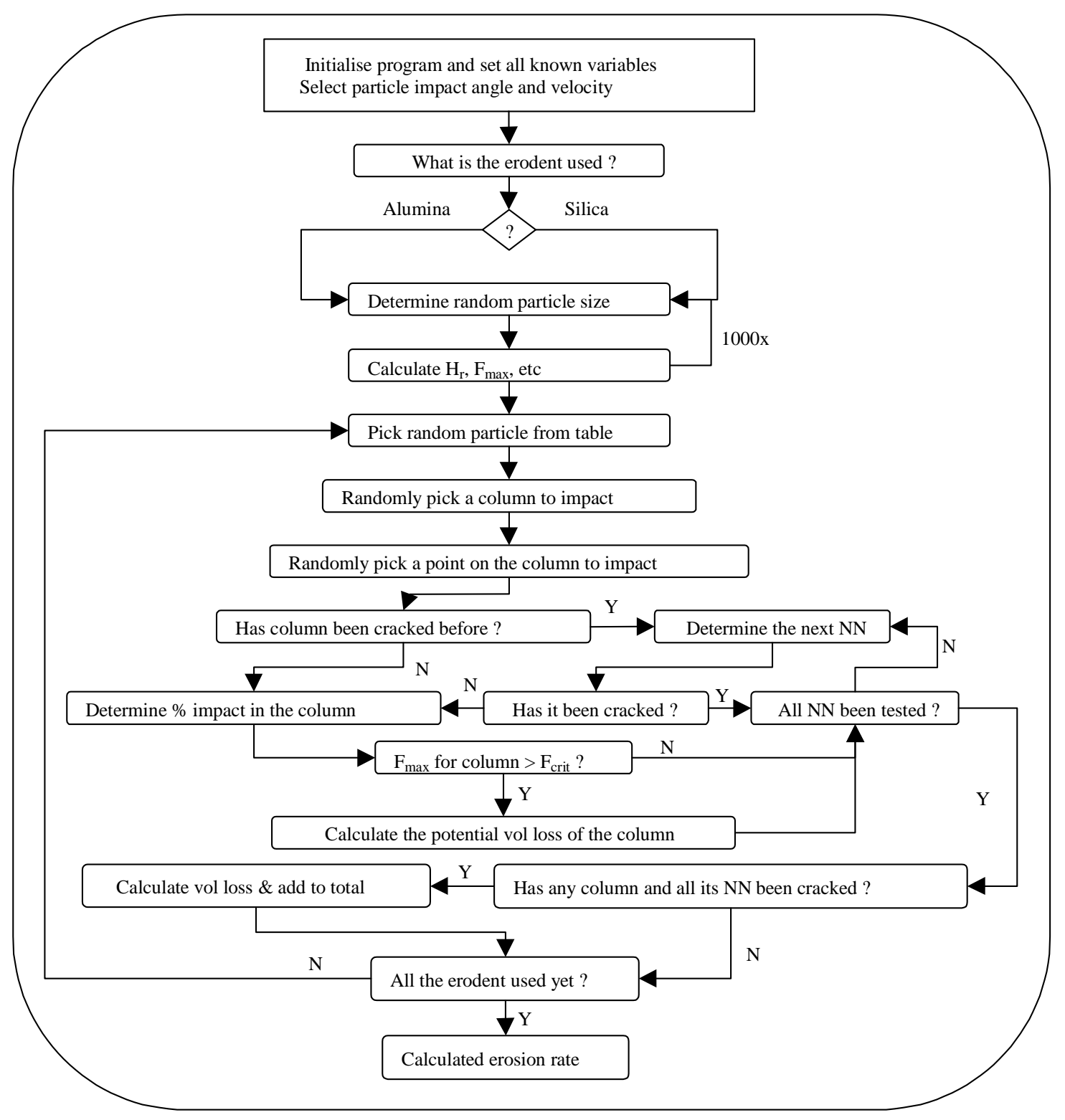

Figure 3: Flow diagram for the EB PVD TBC erosion model. Where ' $\mathrm{H}_{\mathrm{r}}$ ' is the contact radius, ' $\mathrm{F}_{\max }$ ' is the maximum impacting force of the particle, ' $\mathrm{F}_{\text {crit }}$ ' is the critical force necessary to initiate cracking in the column and ' $\mathrm{NN}$ ' is the nearest neighbour. 


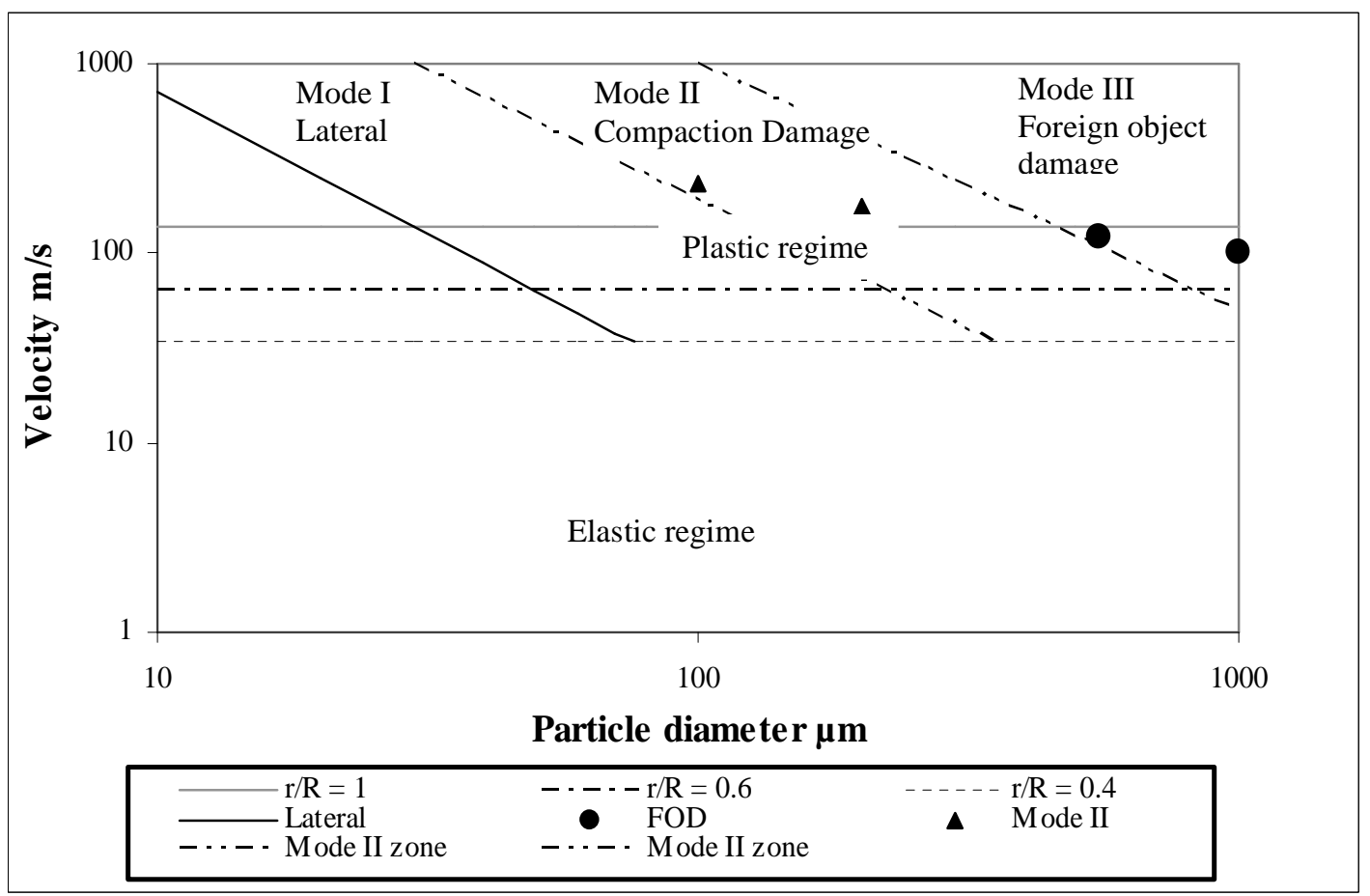

Figure 4: Erosion map for EB PVD TBC's eroded with silica. 


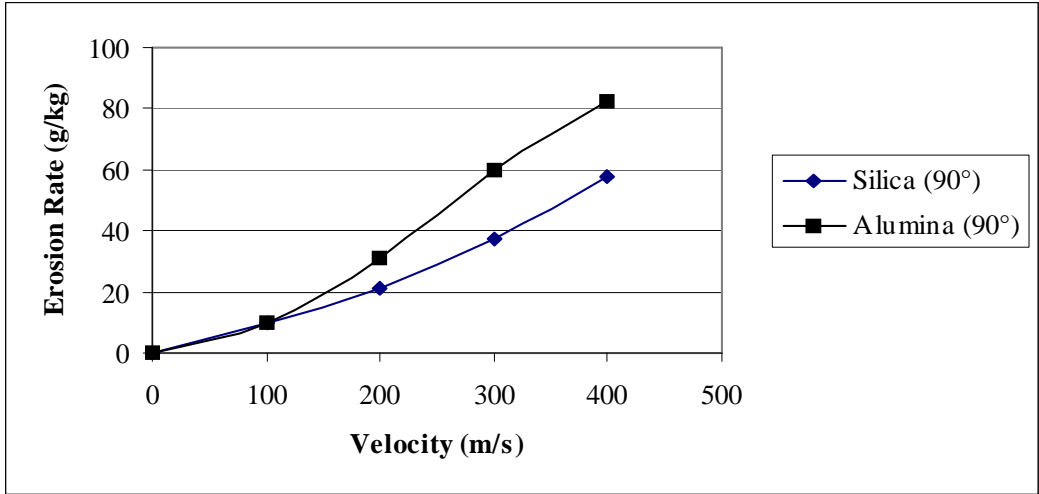

Figure 5: Effect of impact velocity on the erosion rate of EB PVD TBCs. 


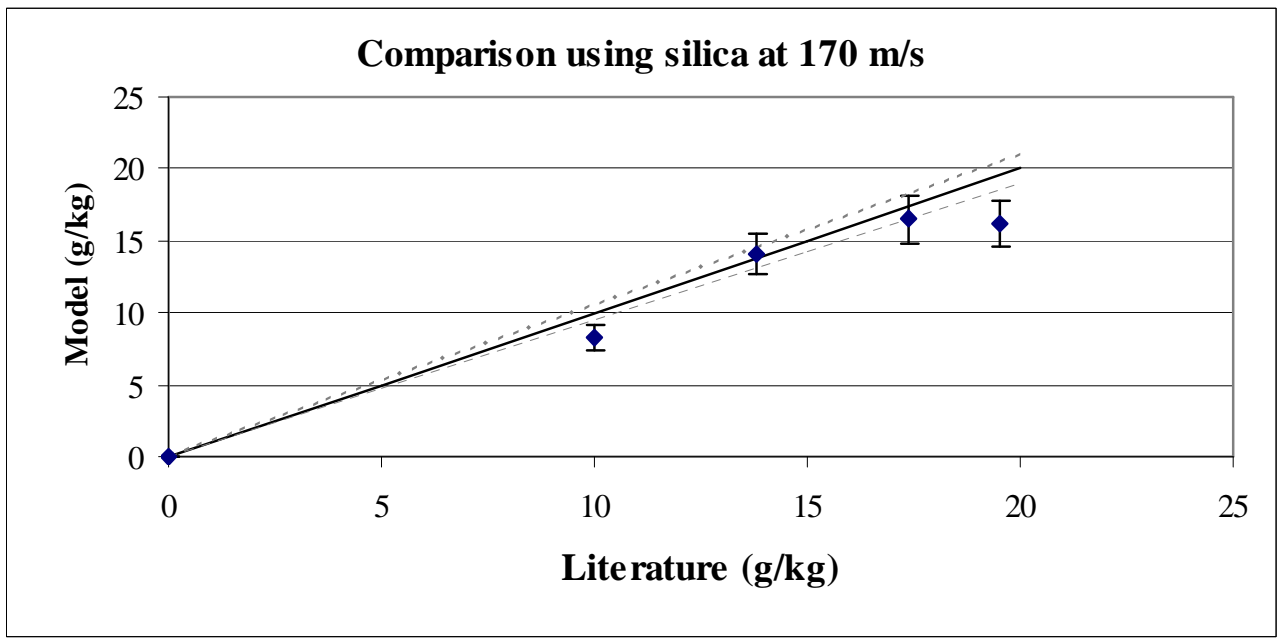

Figure 6: Graph showing the comparison between predicted and measured [6] erosion rates (the superimposed line is drawn for a direct comparison the dashed lines represent one standard deviation). 


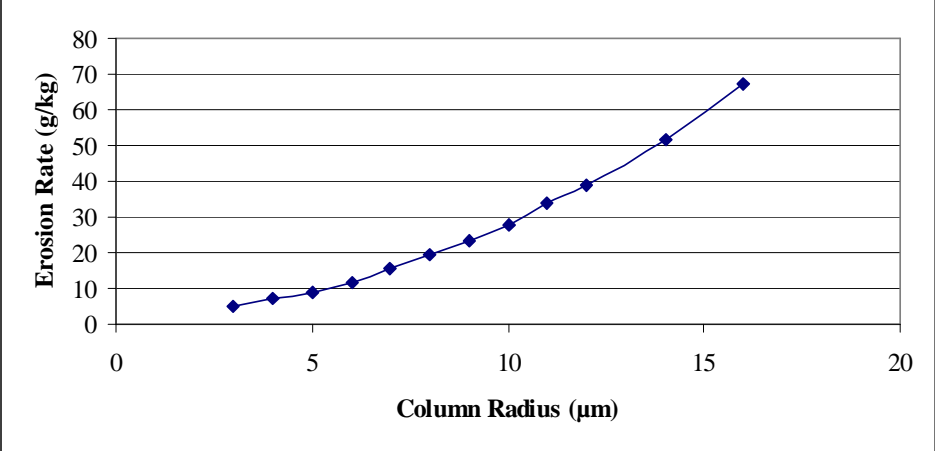

Figure 7: The effect of column diameter on the erosion rate of EB PVD TBCs eroded with silica. 


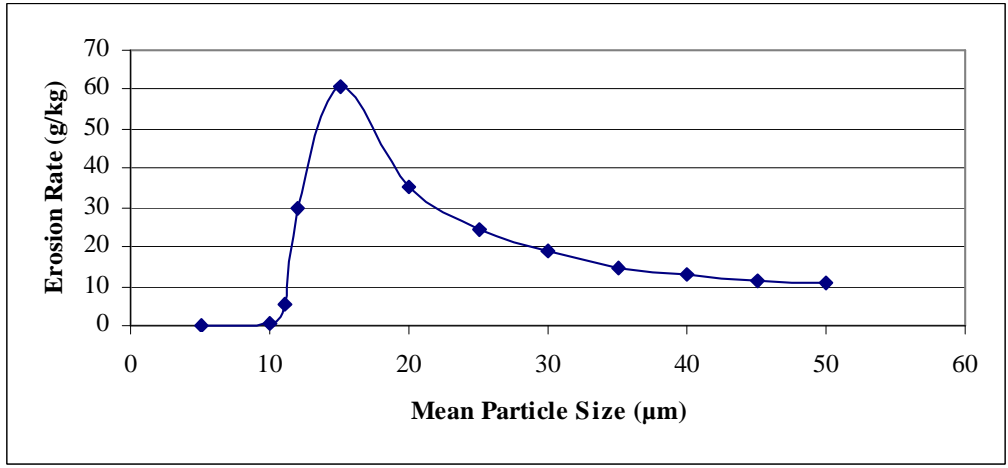

Figure 8: Effect of particle size on the erosion rate of EB PVD TBCs eroded with silica. 


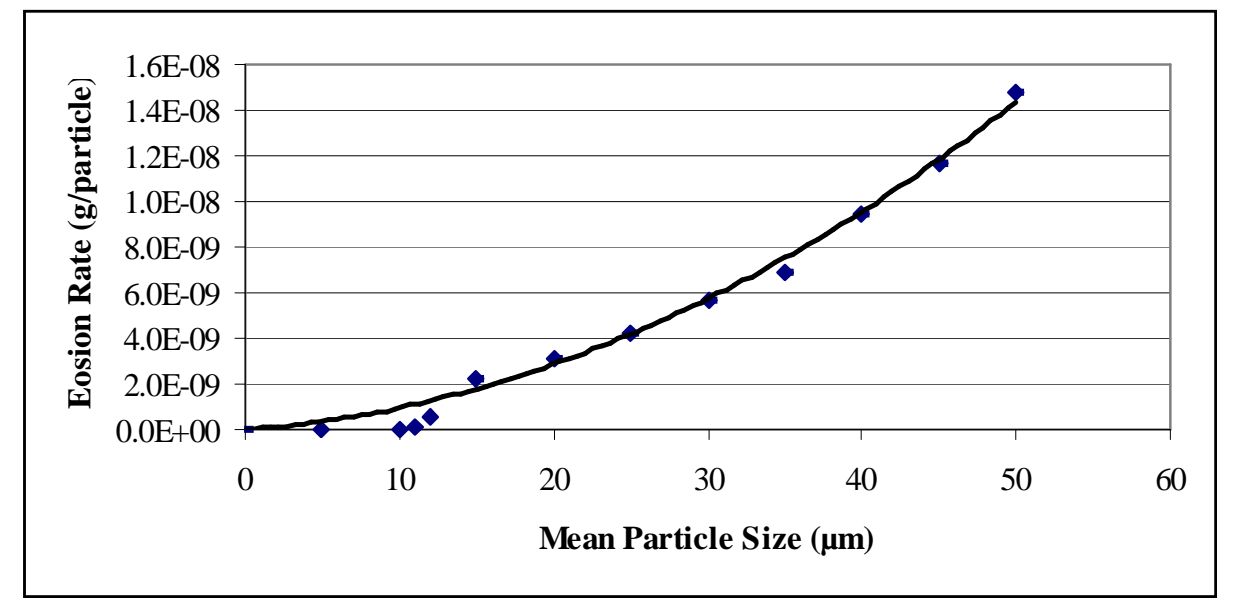

Figure 9: Erosion rate as a function of particle size. 


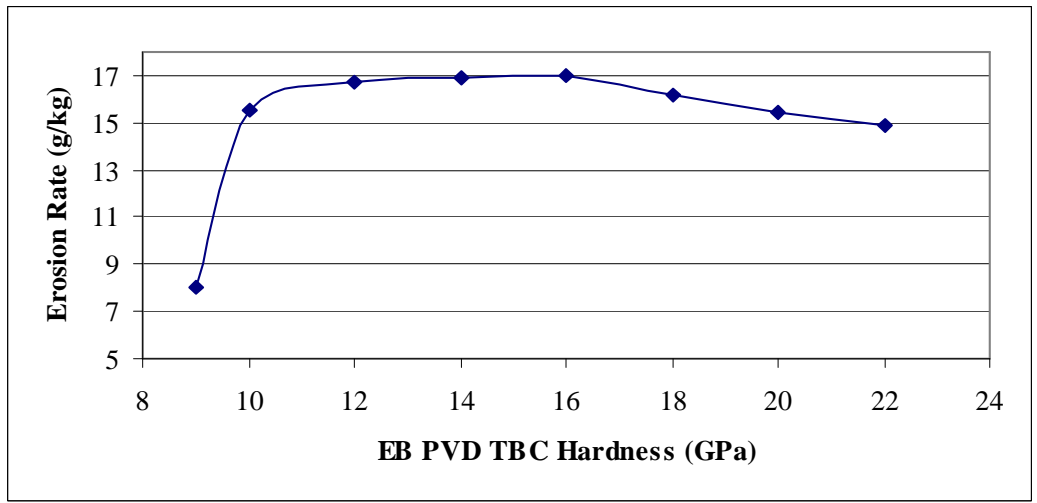

Figure 10: Effect of coating hardness on the erosion rate. 